\title{
Polydiacetylene-Liposome Microarrays for Selective and Sensitive Mercury(II) Detection
}

\author{
By Jiseok Lee, Hayeon Jun, and Jinsang Kim*
}

Conjugated polymers (CPs) are emerging materials for many useful applications. One of the more attractive applications of CPs is for sensor design, due to the signal amplification property and the versatility in molecular design of CPs. ${ }^{[1]}$ Many interesting conjugated-polymer sensors for the detection of chemical and biological molecules have been developed. ${ }^{[2]}$ Polydiacetylene (PDA) is a unique conjugated polymer showing the well-known pressure-sensitive color change. The color change is believed to appear from the conformational change of the conjugated backbone of PDA induced by external stimuli, such as heat, $\mathrm{pH}$ change, and mechanical pressure. ${ }^{[3]}$ Topochemical polymerization through $254 \mathrm{~nm}$ UV irradiation is the most commonly used method to polymerize well-packed diacetylene monomers with a high degree of spatial order, such as that found in LangmuirBlodgett monolayers and self-assembled liposome bilayers. ${ }^{[4]}$ The photoinduced topochemical polymerization converts transparent diacetylene monomers into conjugated PDA with a blue color (absorption $\lambda_{\max }$ at $\approx 640 \mathrm{~nm}$ ). Upon external stimuli, the absorption $\lambda_{\max }$ shifts from $640 \mathrm{~nm}$ (blue phase) to $540 \mathrm{~nm}$ (red phase). Interestingly, the triggered red phase of PDA is also weakly fluorescent, so PDA can provide dual signaling capability. ${ }^{[5]}$ The pressure-sensitive mechanochromism of PDAs has been used for colorimetric biosensor development to detect influenza virus, E. coli, microorganisms, cholera toxin, glucose, and nucleic acids, because shape changes of biological molecules accompany the recognition event. ${ }^{[6]}$

We previously developed PDA-based self-signaling microarrays capable of highly selective, sensitive, and quantitative potassium detection. ${ }^{[7]}$ We also developed emissive PDA nanoparticles with dual signaling capability for sensitive and selective immuno-

[*] Prof. J. Kim, J. Lee

Macromolecular Science and Engineering

University of Michigan

Ann Arbor, MI 48109 (USA)

E-mail: jinsang@umich.edu

Prof. J. Kim, H. Jun

Department of Material Science and Engineering

University of Michigan

Ann Arbor, MI 48109 (USA)

Prof. J. Kim

Chemical Engineering

University of Michigan

Ann Arbor, MI 48109 (USA)

Prof. J. Kim

Biomedical Engineering

University of Michigan

Ann Arbor, MI 48109 (USA)

DOI: 10.1002/adma.200900639 fluorescence labeling. ${ }^{[8]}$ However, the implemented PDA design principle for the PDA-based biosensors is only suitable for a specific single target molecule, because the sensory PDA films and liposomes were prepared from diacetylene monomers with a fixed particular receptor unit. To detect another analyte, a new diacetylene monomer with a new receptor unit has to be synthesized and purified stringently. However, to achieve a PDA microarray with high throughput capacity, various receptors must be present at the microarray surface. This requires efficient tethering of receptors after the liposome immobilization on a glass substrate for both convenience and to allow for user-prepared microarrays, because in many cases researchers will want to put their own receptors, for example, various proteins, on the chip surface. In this regard, the realization of a universal PDA platform will require the development of a novel molecular-design principle that will allow for the incorporation of various receptor units after preparation of sensory PDA films.

In this contribution, we present our recent development of PDA microarrays for selective and sensitive mercury detection. Mercury $\left(\mathrm{Hg}^{2+}\right)$ is a well-known neurotoxin, and its accumulation in the human body induces critical brain damage, resulting in blindness, deafness, memory loss, and death. ${ }^{[9]}$ Therefore, the allowable concentration of mercury in drinking water is strictly regulated to be less than $2 \mathrm{ppb} .^{[10]}$ To detect mercury, various methods have been developed by means of gold nanoparticles, fluorophores, DNAzymes, proteins, and polymers. ${ }^{[11]}$ Our sensory PDA microarrays were developed based on selfassembling diacetylene molecules having an epoxy group to achieve a universal PDA platform for convenient post tethering of receptors. We chose the epoxy group because it can be a versatile functional group for bioconjugation with biological molecules by means of its reaction with ubiquitously present amine groups on biological molecules. Epoxy groups are also stable for storage. ${ }^{[12]}$ $\mathrm{N}$-hydroxysuccinimide (NHS)-activated carboxylic acid is a common choice for immobilization of PDA liposomes on an amine-modified substrate, and an amine is for an aldehydemodified substrate. ${ }^{[7,13]}$ We compared the immobilization efficiency and the stability of the epoxy group with those of NHS-activated carboxylic acid and ethylenediamine.

We synthesized PCDA (10,12-pentacosadiynoic acid)-Epoxy according to the procedure described in the Supporting Information. The self-assembled diacetylene liposomes (Epoxy liposome) were prepared using a 4:1 mixture of the PCDA-Epoxy and PCDA. The two control liposomes, composed of a 1:1 mixture of PCDA:PCDA-linker-NHS (NHS liposome) and 1:1 mixture of PCDA-EDEA:PCDA-EDA (EDA liposome), were prepared according to the literature procedures. ${ }^{[7,13]}$ We immobilized the Epoxy and NHS liposomes on an amine-modified glass substrate and the EDA liposome on an aldehyde-modified glass substrate, 
respectively, using identical conditions. The results showed that PCDA-Epoxy liposomes have much faster immobilization kinetics, and result in better film quality and better stability than the other two (the detailed results are in the Supporting Information).

We developed highly selective and sensitive PDA microarrays for mercury detection based on the Epoxy liposome system developed. Scheme 1 illustrates our design strategy. Note that the same epoxy units were used for liposome tethering onto the substrate and for the post-tethering of the ssDNA aptamer as a selective receptor for mercury detection. Studying the literature, we identified the thymine-rich ssDNA aptamer (5'-TTCTTTCTTCCCCTTGTTTGTT- $\left.{ }^{\prime}\right)$ that forms a thymine- $\mathrm{Hg}^{\mathrm{II}}$-thymine complex (T-Hg-T) by selective binding with $\mathrm{Hg}^{2+} \cdot{ }^{14]}$ As schematically illustrated in Scheme 1, our PDA mercury sensors are designed in such a way that when the ssDNA aptamers recognize and wrap around mercury ions, the resulting bulky T-Hg-T complexes repulse each other. The static repulsion force is then transferred to the PDA liposomes to perturb their conjugated ene-yne backbone, and produces the color change from blue to red and the red fluorescence emission. The PCDA-Epoxy/PCDA (4:1) liposome solution was first immobi- lized onto an amine glass slide. We then spotted the thymine-rich ssDNA aptamer using a microarrayer onto the liposome layer and incubated the slide at room temp for $3 \mathrm{~h}$ under $70 \%$ humidity to prevent the aptamer solution from drying out. After rinsing away any unreacted ssDAN aptamers, we photopolymerized the liposome slide using a $254 \mathrm{~nm}$ UV lamp $\left(1 \mathrm{~mW} \mathrm{~cm}^{-2}\right)$ for $20 \mathrm{~s}$.

First, we tested the sensitivity of the PDA microarray. Figure 1 shows the fluorescence microscopy images of the PDA microarray after incubation with $\mathrm{Hg}^{2+}$ solution in various concentrations at room temperature for $1 \mathrm{~h}$. The developed red-fluorescence intensity has a close relationship with the concentration of the mercury solution, and the correlation is shown in Figure 1D. As the concentration of mercury ions increases, more T-Hg-T complexes will be formed, and induce stronger perturbation of the ene-yne backbone of the PDA liposomes, resulting in the increase in the red fluorescence intensity. Based on the correlation curve, therefore, a quantitative analysis of an unknown mercury concentration is also achievable. The detection limit after $1 \mathrm{~h}$ of incubation confirmed by microscopy images was $0.005 \mathrm{~mm}(0.027 \mathrm{mg} / 20 \mathrm{~mL})$. This detection limit is imposed by our microscope, and could be much better if a more sensitive equipment was used.

A)
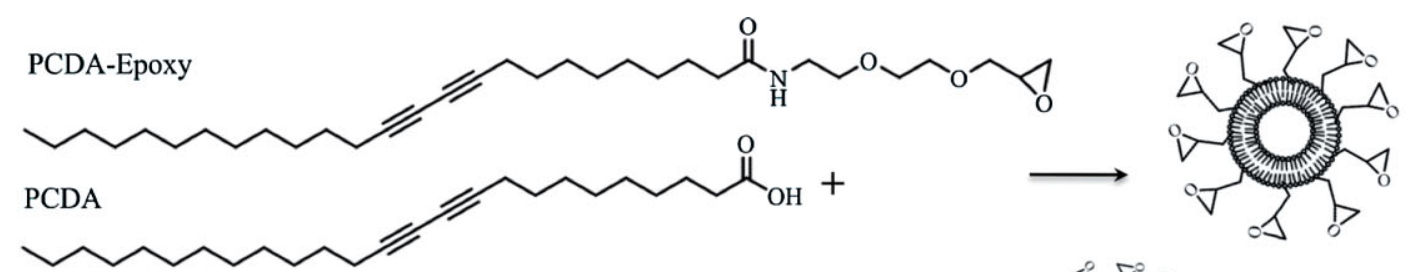

B)
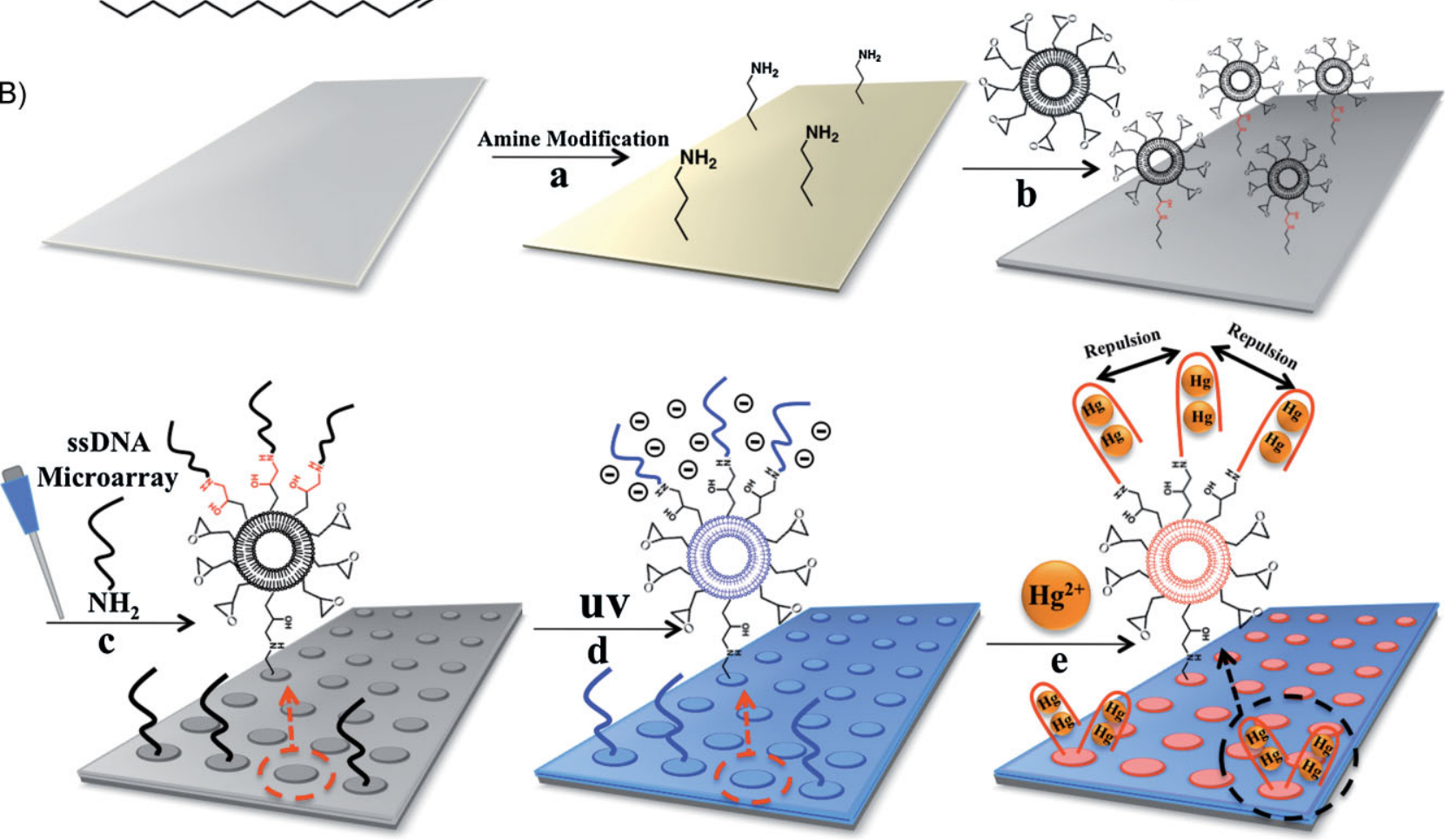

Scheme 1. A) Chemical structure of the diacetylene monomers, PCDA and PCDA-Epoxy. B) Schematic illustration of the PDA liposome-based microarray for mercury detection. a) Surface modification of the glass substrate with amine functionality. b) Immobilization of the Epoxy liposomes onto the amine glass slide through epoxy-amine coupling. c) Post-tethering of the ssDNA aptamer by means of a microarrayer. d) Photopolymerization of the PDA liposomes using a $254 \mathrm{~nm}$ UV lamp. e) Recognition of the target mercury ions results in red fluorescent emission. 

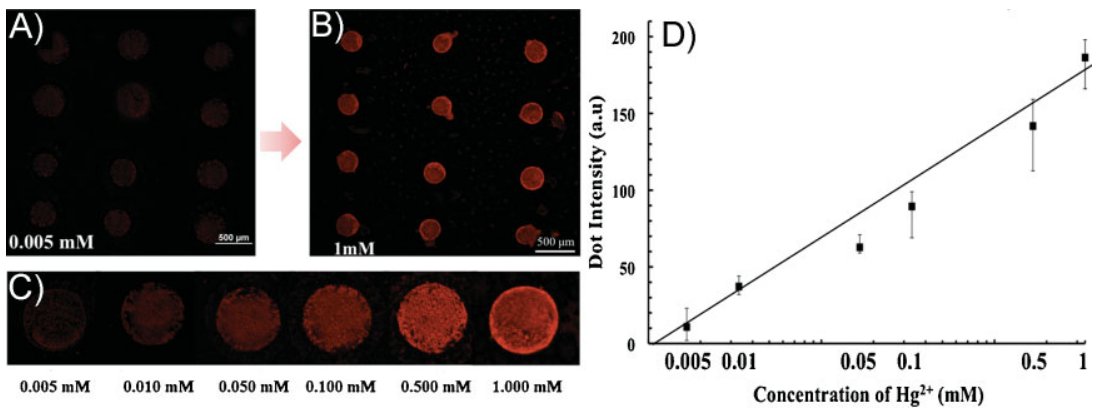

Figure 1. Fluorescence microscopy images of the PDA microarray (excitation at $600 \mathrm{~nm}$ and a long-pass emission filter with $550 \mathrm{~nm}$ cutoff were used) after $1 \mathrm{~h}$ incubation at room temperature with A) $0.005 \mathrm{~mm}$ of $\mathrm{Hg}^{2+}$ and B) $1 \mathrm{~mm}$ of $\mathrm{Hg}^{2+}$ solution. C) Fluorescence microscopy images of the PDA liposome arrays after $1 \mathrm{~h}$ incubation at room temperature with $\mathrm{Hg}^{2+}$ solutions in various concentrations. Scale bar is $500 \mu \mathrm{m}$. D) Correlation curve between the fluorescence intensity and the amount of $\mathrm{Hg}^{2+}$.
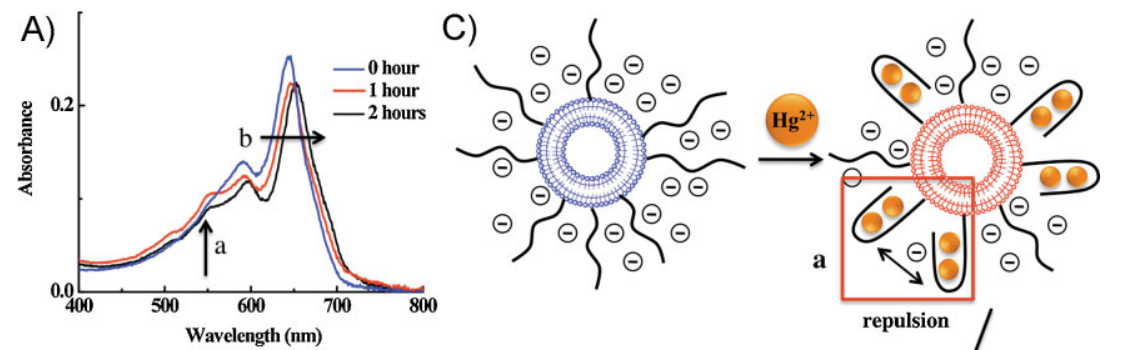

B)
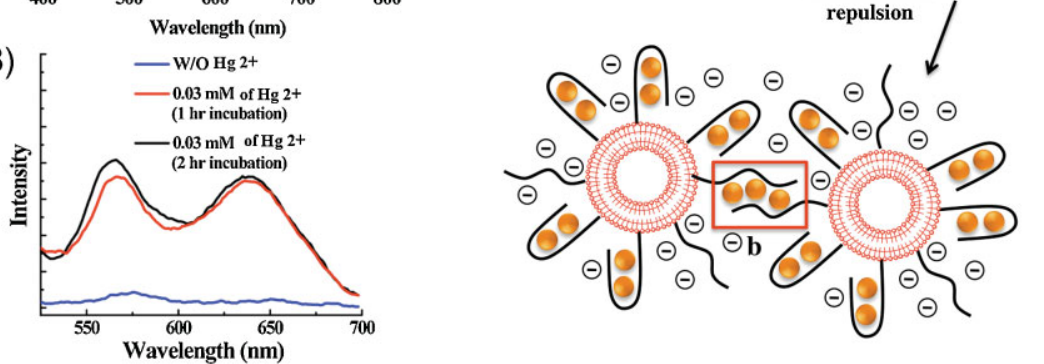

Figure 2. A) UV-vis spectra and B) PL spectra of the PDA liposome solution (0.05 mm) upon addition of $\mathrm{Hg}^{2+}(0.03 \mathrm{~mm})$ for $1 \mathrm{~h}$ (red line) and $2 \mathrm{~h}$ (black line) of incubation. C) Schematic illustration of the T-Hg-T conformation in the PDA liposome solution: a) the resulting steric repulsion between the intermolecular $\mathrm{T}-\mathrm{Hg}$ - $\mathrm{T}$ complexes after $1 \mathrm{~h}$ incubation at room temperature and $\mathrm{b}$ ) the formation of the intermolecular $\mathrm{T}-\mathrm{Hg}$-T aggregation after $2 \mathrm{~h}$ incubation at room temperature.
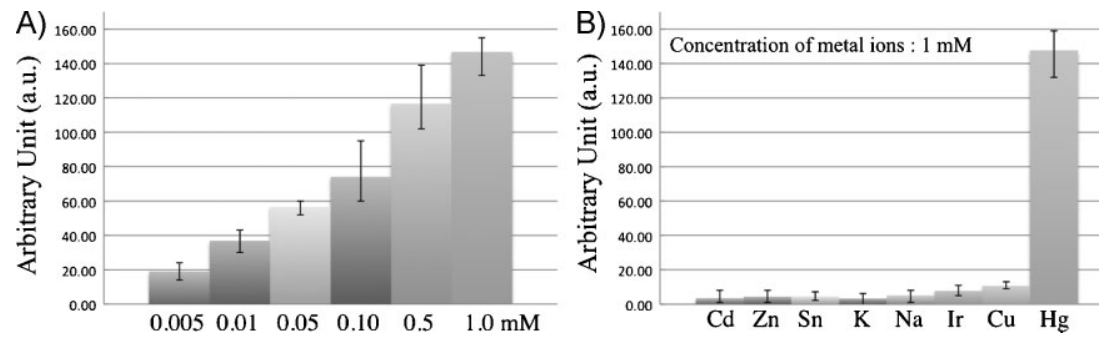

Figure 3. A) Fluorescence intensity of the PDA microarray after $1 \mathrm{~h}$ incubation with $\mathrm{Hg}^{2+}(0.005$, $0.01,0.05,0.10,0.50,1.00 \mathrm{~mm})$. B) Fluorescence intensity of the PDA microarray after $1 \mathrm{~h}$ incubation with each $1.0 \mathrm{~mm}$ metal ion.
We also conducted a detection study with the PDA liposome in solution to better understand the recognition of $\mathrm{Hg}^{2+}$ by the ssDNA aptamer. Figure 2 shows the UV-vis absorption and PL emission spectra of the PDA-liposome solution upon addition of mercury $\left(\mathrm{Hg}^{2+}\right)$ ions at room temperature. The absorption peak at $650 \mathrm{~nm}$ decreased and the red-phase absorption band appeared upon addition of $0.03 \mathrm{mM}$ of $\mathrm{Hg}^{2+}$ (Fig. 2A). The fluorescence intensity of the PDA liposomes also increased upon addition of $\mathrm{Hg}^{2+}$ (Fig. 2B). Interestingly, after $2 \mathrm{~h}$ incubation, the UV-vis spectrum showed a bathochromic shift, as shown in Figure 2A, and the PDA liposomes formed aggregation. Unlike the PDA liposomes in the solid microarray, the PDA liposomes in solution have freedom of translational movement. Therefore, the thymine-rich ssDNA liposome in solution can form intermolecular as well as intramolecular T-Hg-T complexes, as schematically illustrated in Figure 2C. The formation of the intermolecular T-Hg-T complexes is likely the origin of the observed aggregation and the slight bathochromic shift in UV-vis spectrum.

We investigated the selectivity of the PDA microarray by incubating various metal ions, such as $\mathrm{Cd}^{2+}, \mathrm{K}^{+}, \mathrm{Na}^{+}, \mathrm{Sn}^{2+}, \mathrm{Ir}^{2+}, \mathrm{Cu}^{2+}$, and $\mathrm{Zn}^{2+}$, with the PDA microarray. As can be seen in Figure $3 \mathrm{~A}$ and $\mathrm{B}$, the fluorescence-emission intensity induced by other metal ions are orders of magnitude smaller than that induced by the same concentration of mercury ion, demonstrating the excellent selectivity of the PDA-liposome array.

In summary, we have developed PDAliposome-based microarrays for selective and sensitive mercury detection. We investigated and identified the epoxy group as a universal functional group for efficient immobilization of PDA liposome onto a solid substrate, and for the convenient post-tethering of the amine-modified ssDNA aptamer (5'-TTCTTTCTTCCCCTTGTTTGTT-3') on the liposome surface as a selective receptor for the recognition of mercury ion. The PDA mercury sensors are designed in such a way that when the ssDNA aptamers recognize and wrap around mercury ions, the steric repulsion between the resulting bulky T-Hg-T complexes perturbs the conjugated ene-yne backbone of the PDA liposomes, and produces the color change from blue to red and the red fluorescence emission. The detection limit of the PDA microarray is $5 \mu \mathrm{M}$. The specificity of the ssDNA aptamer toward $\mathrm{Hg}^{2+}$ provides excellent selectivity to the PDA microarray as well. The developed Epoxy-based PDA- 
liposome design is an excellent universal PDA platform that can be readily applicable to other sensor designs, allowing fast formation of the PDA-liposome layer and efficient tethering of receptors after the liposome immobilization on a glass substrate for both convenience and to allow for user-prepared microarrays.

\section{Experimental}

Preparation of the Liposomes: A mixture of the PCDA-Epoxy and PCDA (4:1 mole ratio) was dissolved in $0.2 \mathrm{~mL}$ of tetrahydrofuran. The solvent mixture was injected into $5 \mathrm{~mm}$ 4-(2-hydroxyethyl)-1-piperazineethanesulfonic acid (HEPES) buffer at $\mathrm{pH} 8.0(30 \mathrm{~mL})$ and bath-sonicated for $5 \mathrm{~min}$ to produce the final concentration of the lipid vesicle of $0.5 \mathrm{~mm}$. After the sonication, the solution was filtered through $0.8 \mu \mathrm{m}$ cellulose syringe filter three times to remove liposomes of undesired size, and stored at $5{ }^{\circ} \mathrm{C}$ for $2 \mathrm{~h}$.

Immobilization of the Liposomes: An amine-modified glass slide was incubated in the PCDA-Epoxy/PCDA(4:1) liposome solution for $20 \mathrm{~min}$ at room temperature. The PDA-liposome-immobilized glass slide was vigorously rinsed using $10 \mathrm{~mm}$ HEPES buffer $\mathrm{pH} 8.0$ for $3 \mathrm{~min}$. The glass was dried and stored under nitrogen at $5^{\circ} \mathrm{C}$. The immobilization of the PCDA-EDEA/PCDA-EDA(1:1) was carried out as described in ref. [12, and the PCDA-linker-NHS/PCDA(1:1) liposome was immobilized according to the procedures in ref. [7].

Fabrication of the PDA Microarray: A solution of $100 \mu \mathrm{m}$ of ssDNA in $5 \mathrm{~mm}$ HEPES buffer $\mathrm{pH} 9.5 / 3 \times$ SSC Buffer $(1: 1)$ was prepared. The ssDNA probe solution was then heated at $90^{\circ} \mathrm{C}$ for $3 \mathrm{~min}$. Thymine-rich ssDNA was spotted onto the glass slide coated with the PDA liposomes using a manual microarrayer (VP 475,V\&P scientific, INC) at 70\% humidity. The glass slide with the spotted ssDNA was incubated for $6 \mathrm{~h}$ at $75 \%$ humidity. After rinsing with 1\% SDS buffer $\mathrm{pH} 8.0$ for $3 \mathrm{~min}$ and deionized water, the slide was dried under a stream of nitrogen followed by the photopolymerization with $254 \mathrm{~nm}$ UV light $\left(1 \mathrm{~mW} \mathrm{~cm}{ }^{-2}\right)$ for $20 \mathrm{~s}$.

\section{Acknowledgements}

This research was supported by NSF CAREER Award (DMR 0644864), and the Center for Chemical Genomics-Thermo Fisher Grant. Supporting Information is available online from Wiley InterScience or from the author.

Received: February 23, 2009 Revised: March 26, 2009 Published online: May 21, 2009

[1] a) T. M. Swager, Acc. Chem. Res. 1998, 31, 201. b) M. Lerclerc, Adv. Mater. 1999, 11, 1491. c) D. T. McQuade, A. E. Pullen, T. M. Swager, Chem. Rev. 2000, 100, 2537. d) S. W. Thomas, III, G. G. Joly, T. M. Swager, Chem. Rev. 2007, 107, 1339.

[2] a) J.-S. Yang, T. M. Swager, J. Am. Chem. Soc. 1998, 120, 5321. b) J. Kim, D. T. McQuade, S. K. Mchugh, T. M. Swager, Angew. Chem. Int. Ed. 2000, 39,
3868. c) R. Sakai, I. Otsuka, T. Satoh, R. Kakuchi, H. Kaga, T. Kauchi, Macromolecules 2006, 39, 4032. d) B. Liu, G. C. Bazan, Chem. Mater. 2004, 16, 4467. e) H. A. Ho, M. Biissinot, M. G. Bergeron, G. Corbeil, K. Dore, D. Boudreau, M. Leclerc, Angew. Chem. Int. Ed. 2002, 41, 1548. f) C.-C. Pun, K. Lee, H. J. Kim, J. Kim, Macromolecules 2006, 39, 7461. g) K. Lee, L. K. Povlich, J. Kim, Adv. Funct. Mater. 2007, 17, 2580. h) K. Lee, K. Maisel, J.-M. Rouillard, E. Gulari, J. Kim, Chem. Mater. 2008, 20, 2848. i) K. Lee, J.-M. Rouillard, T. Pham, E. Gulari, J. Kim, Angew. Chem. Int. Ed. 2007, 46, 4667. j) Y. Liu, K. S. Schanze, Anal. Chem. 2008, 80, 8605. k) I. B. Kim, U. H. F. Bunz, J. Am. Chem. Soc. 2006, 128, 2818.

[3] a) J. B. Pang, L. Yang, B. McCaughey, H. S. Peng, H. S. Ashbaugh, C. J. Brinker, Y. F. Lu, J. Phys. Chem. B 2006, 110, 7221. b) R. W. Carpick, T. M. Mayer, D. Y. Sasaki, A. R. Burns, Langmuir 2000, 16, 4639. c) K. Morigaki, T. Baumgart, U. Jonas, A. Offenhausser, W. Knoll, Langmuir 2002, 18, 4082. d) Q. Cheng, R. C. Stevens, Langmiur 1998, 14, 1974. e) R. W. Carpick, D. Y Sasaki, A. R. Burns, Langmiur 2000, 16, 1270. e) G. Leiser, B. Tieke, G. Wegner, Thin Solid Films 1980, 68, 77.

[4] a) G. Wegner, Z. Naturforsch. B 1969, 24, 824. b) D. H. Charych, J. O. Nagy, W. Spevak, M. D. Bednarski, Science 1993, 261, 585. c) Y. Okawa, M. Aono, Nature 2001, 409, 683. d) A. Miura, S. D. Feyter, M. M. S. Abdel-Mottaleb, A. Gesquière, P. C. M. Grim, G. Moessner, M. Sieffert, M. Klapper, K. Müllen, F. C. De Schryver, Langmiur 2003, 19, 6474.

[5] a) R. R. Chance, Macromolecules 1980, 13, 396. b) J.-M. Kim, J.-S. Lee, H. Choi, D. Sohn, D. J. Ahn, Macromolecules 2005, 38, 9366.

[6] a) A. Reichert, J. O. Nagy, W. Spevak, D. Charych, J. Am. Chem. Soc. 1995 117, 829. b) J. Song, Q. Cheng, R. C. Stevens, Chem. Phys. Lipids 2002, 114, 203. c) Q. Cheng, R. C. Stevens, Adv. Mater. 1997, 9, 481. d) J.-M. Kim, J.-S. Lee, J.-S. Lee, S. Woo, D. J. Ahn, Macromol. Chem. Phys. 2005, 206, 2299. e) Y. K. Jung, T. W. Kim, C. Jung, D. Cho, H. G. Park, Small. 2008, 4, 1778. f) M. A. Reppy, B. A. Pindzola, Chem. Commun. 2007, 4317. g) D. J. Ahn, J.-M. Kim, Acc. Chem. Res. 2008, 41, 805.

[7] J. Lee, H. Kim, J. Kim, J. Am. Chem. Soc. 2008, 130, 5010.

[8] H. Kim, J. Lee, T. Kim, T. S. Lee, J. Kim, Adv. Mater. 2008, 20, 1117.

[9] a) W. Zheng, M. Aschner, J.-F. Ghersi-Egea, Toxicol. Appl. Pharmacol. 2003, 192, 1. b) D. P. Wojcik, M. E. Godfrey, D. Christie, B. E. Haley, Neuroendocrinol. Lett. 2006, 27, 415. c) J. Mutter, J. Naumann, R. Schneider, H. Walach, B. Haley, Neuroendocrinol. Lett. 2005, 26, 439.

[10] Environmental Standards for Mercury, Background Information on Mercury Sources and Regulations, United State Environmental Protection Agency (EPA), www.epa.gov.

[11] a) J.-S. Lee, M. S. Han, C. A. Mirkin, Angew. Chem. Int. Ed. 2007, 46, 4093 b) C.-C. Huang, C.-K. Ching, Z.-H. Lin, K.-H. Lee, H.-T. Chang, Anal. Chem. 2008, 80, 1497. c) T. Li, S. Dong, E. Wang, Anal. Chem. 2009, 81, 2144. d) P. Chen, C. He, J. Am. Chem. Soc. 2004, 126, 728. e) Y. Zhao, Z. Zhong, J. Am. Chem. Soc. 2006, 128, 9988.

[12] C. Mateo, 1 V. Grazu, J. M. Palomo, F. Lopez-Gallego, R. Fernandez-Lafuente, J. M. Guisan, Nat. Protocols 2007, 2, 1022.

[13] a) J.-M. Kim, Y. B. Lee, D. H. Yang, J.-S. Lee, G. S. Lee, D. J. Ahn, J. Am. Chem. Soc. 2005, 127, 17580. b) J.-M. Kim, E.-K. Ji, S.-M. Woo, H. Lee, D. J. Ahn, Adv. Mater. 2003, 15, 1118.

[14] a) X. Liu, Y. Tang, L. Wang, J. Zhang, S. Song, C. Fan, S. Wang, Adv. Mater. 2007, 19, 1471. b) A. Ono, H. Togashi, Angew. Chem. Int. Ed. 2004, 43, 4300. c) X.-J. Zhu, S.-T. Fu, W.-K. Wong, J.-P. Guo, W.-Y. Wong, Angew. Chem. Int. Ed. 2006, 45, 3150. 